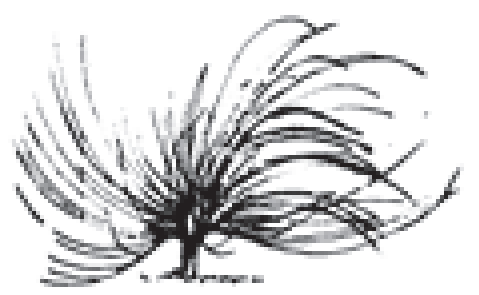

\title{
Educación Superior y Estudiantes Indígenas en Oaxaca: Problemas y Desafíos
}

\author{
Omar de Jesús Reyes Pérez ${ }^{1}$ \\ Universidad del Mar \\ Santa María Huatulco, Oaxaca, México \\ omar@huatulco.umar.mx \\ Noemí López Santiago ${ }^{2}$ \\ Universidad del Mar \\ Santa María Huatulco, Oaxaca, México \\ noemi@huatulco.umar.mx
}

\begin{abstract}
Resumen
Los alumnos universitarios provenientes de grupos indígenas se enfrentan a desafíos particulares que se agregan a los retos propios de cursar una carrera universitaria. Por si esto fuera poco, estudiar una carrera en uno de los estados más pobres y con menor nivel académico del país supone esfuerzos extraordinarios por parte de los alumnos para llevar a buen término su carrera. En este trabajo se presentan algunos de estos retos de estudiantes indígenas que estudian en la Universidad del Mar, Campus Huatulco.
\end{abstract}

Palabras clave: universidad, indígena, Oaxaca, problemas, desafios.

Recibido: 11 de abril de 2014-Aprobado: 25 de octubre de 2014

1 Profesor-investigador de tiempo completo en la Universidad del Mar, Instituto de Estudios Internacionales, Maestro en Filosofía por la Universidad Nacional Autónoma de México.

2 Profesora-investigadora de tiempo completo en la Universidad del Mar, Instituto de Estudios Internacionales, Maestra en Economía por la Universidad Autónoma de Coahuila. 


\begin{abstract}
College students from Indigenous groups face particular challenges that add up to the challenges of taking a university major. As if this were not enough, styudying a major in one of the poorest Mexican states and with a lower academic level in the country poses extraordinary efforts to the students to complete their majors successfu1ly. This paper presents some of the challenges faced by indigenous students studying at "Universidad del Mar," Huatulco Campus.
\end{abstract}

Keywords: university, indigenous people, Oaxaca, problems, challenges

\title{
Introducción
}

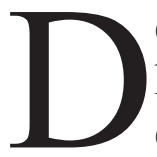

e acuerdo con los datos del Censo de Población y Vivienda, hasta el año 2010 hay un total de 3, 801, 962 habitantes en el estado de Oaxaca. ${ }^{3}$ La población indígena de 15 años y más representa el $44.37 \%$ del total de la población, de ahí el $24.6 \%$ es analfabeta y el $20.7 \%$ no tiene ningún nivel de instrucción y se encuentran divididos en 16 grupos etnolingüísticos.

Lo anterior revela la carencia y los retos que se tiene en el estado de Oaxaca en materia de educación en sus distintos niveles (básico, medio superior y superior), tomando en cuenta que el nivel promedio de escolaridad es de 6.9 años, por debajo del promedio nacional que corresponde a 8.6 años.

No obstante, que la educación es considerada como una herramienta que tiene una poderosa fuerza de cambio y transformación positiva, es importante reconocer que en ocasiones, las instituciones académicas de nivel superior, se encargan únicamente de reproducir reglas, normas, costumbres y valores que muy poca relación tienen con el objetivo de proporcionar una formación integral que ayude a resolver las necesidades de la realidad indígena.

3 El estado de Oaxaca se encuentra ubicado en la parte sur del país. Colinda al norte con los estados de Puebla y Veracruz; al este con Chiapas; al sur con el Océano Pacífico; al oeste con Guerrero. Es el estado con mayor número de municipios en todo el país pues cuenta con 570, distribuidos en ocho regiones: Mixteca, Cañada, Sierra Norte, Papaloapan, Sierra Sur, Valles Centrales, Costa e Istmo. 
Desde siempre se ha privado a la población indígena de educación de calidad. Los pocos espacios educativos a los que se tiene acceso no han sido suficientes ni adecuados a las necesidades que se requieren, por lo que el acceso a la educación superior para estudiantes indígenas es muy difícil considerando las desventajas sociales, económicas y académicas.

Generalmente, los estudiantes indígenas no son objeto de estudio aun cuando estos representan una parte fundamental del sistema educativo a nivel superior. Por ello, el objetivo del presente texto es conocer e identificar los problemas personales, académicos, culturales y sociales a los que se enfrentan los estudiantes indígenas durante su estancia en la Universidad del Mar (UMAR), Campus Huatulco del estado de Oaxaca.

\section{Educación superior para indígenas}

La educación universitaria, tal como la conocemos, es un invento occidental, por lo tanto, para los grupos indígenas representa un gran esfuerzo integrar a su cosmovisión la idea de que tener un título universitario es un valor, sobre todo cuando tienen que abandonar sus comunidades para asistir a un centro escolar, postergando la participación en la productividad familiar.

En el caso de los indígenas o comunidades rurales, la educación formal no tiene un fin práctico relevante, pues lo que se considera éxito social en estos grupos sociales tiene que ver más con las convenciones locales, como podría ser la siembra, la caza, la pesca, la construcción de sus viviendas, en fin, actividades que no requieren de una especialización teórica y sí una participación en estas labores desde edades tempranas.

Por la conformación social, la educación superior, incluso la pública, se puede considerar una educación a la cual no todos pueden acceder y para algunos grupos sociales es simplemente inalcanzable. Aún cuando las cuotas de las universidades públicas son relativamente bajas, el hecho de que un miembro de la familia en edad productiva se ausente por cuatro o más años implica un desgaste en los demás miembros de grupo familiar. A esto hay que añadirle que los lugares con matrícula son limitados por la gran cantidad de alumnos que aspiran a una ficha, generando cada año conflictos porque no hay espacios para todos los concursantes. 
Barreno (2003) señala que, para 1990 "México cuenta con la mayoría de la población indígena del continente... (y) En cuanto a la educación superior (...) las estipulaciones son que la matrícula indígena en el nivel superior es apenas el $1 \%$..." por lo que México registra el número más bajo de estudiantes indígenas a nivel superior en América Latina.

Información de la Secretaría de Educación Pública (SEP) muestra que para el ciclo escolar 2011-2012, la matrícula escolarizada de educación superior a nivel nacional es de 2, 932, 254 alumnos, que equivale al $29.4 \%$ de la población total de 19 a 23 años de edad (sin incluir posgrado).

Por su parte, la Asociación Nacional de Universidades e Instituciones de Educación Superior (ANUIES) a través del Programa de Apoyo a Estudiantes Indígenas en Instituciones de Educación Superior (PAEIIES) reporta para el año 2010, una matrícula de 163, 015 estudiantes indígenas, en educación superior, de un total de 16 estados de la república mexicana. Aunado a ello, la Comisión Nacional para el Desarrollo de los Pueblos Indígenas (CDI) señala que el 70\% de la población indígena de entre los 15 y 24 años no asiste a la escuela, es decir, no accede a la educación media superior y superior.

Particularmente, en el estado de Oaxaca hasta el ciclo escolar 2011-2012, la SEP presenta un registro de 60, 500 alumnos inscritos en educación superior ${ }^{4}$, de los cuales, según el PAEIIES 21, 656 son estudiantes indígenas (cifra que representa apenas al 1.88\% de la población indígena de la entidad de 15 años y más).

De acuerdo con la ANUIES, los estudiantes indígenas que cursan una carrera profesional, enfrentan en su proceso educativo diversos problemas que obstaculizan su estancia, y en algunos casos, la conclusión de su formación profesional: 1) falta infraestructura educativa adecuada en las regiones de origen, lo que conlleva a,2) la necesidad de emigrar a otros estados u otras regiones al interior de sus entidades, 3) dada su condición económica se tienen que incorporar al mercado de trabajo a temprana edad (ante la carencia de becas), 4) perdida de la identidad indígena y comunitaria derivado de la ausencia de programas orientados a la educación intercultural. En este sentido, es pertinente reconocer un proceso constructivista en el sistema enseñanza-aprendizaje.

430,384 son mujeres y 30, 116 son hombres; distribuidos 563 en nivel técnico superior, 6205 en normal licenciatura y 52, 264 licenciatura universitaria y tecnológica. 


\section{Constructivismo}

La educación inicialmente era un esfuerzo por estandarizar los saberes en los individuos que se encontraban en los centros de estudio y la educación superior tenía como meta especializar a los estudiantes en ciertas áreas del conocimiento bajo el supuesto de que contaban con los requisitos básicos adquiridos en los niveles anteriores. Por tanto, ya no era trabajo de las universidades preocuparse por los conocimientos básicos, su trabajo era la especialización y, bajo el supuesto de que los alumnos son adultos, la preocupación por la vida personal era (y sigue siendo en muchos centros académicos) simplemente irrelevante; mientras el alumno mantuviera los estándares requeridos, lo demás no era incumbencia de la institución.

Con el reconocimiento de las necesidades particulares de los alumnos, las universidades en la actualidad han implementado programas de tutorías, así como departamentos de orientación educativa y psicología. Esta situación ha traído como consecuencia que las instituciones tomen conciencia de las necesidades personales de sus estudiantes así como la realidad que hay detrás de una persona sentada en un pupitre.

Tomar en consideración la realidad que circunda a los alumnos es fundamental en el constructivismo, puesto que "el conocimiento no es el resultado de una mera copia de la realidad pre-existente, sino de un proceso dinámico e interactivo a través del cual la información externa es interpretada y reinterpretada por la mente que va construyendo progresivamente modelos explicativos cada vez más complejos" (Garzón y Vivas, 1999). Esa continua reinterpretación solo puede darse en la vida contextualizada del sujeto.

Para entender el proceso constructivista es fundamental conocer los siguientes postulados:

1. El conocimiento no es innato (como en el idealismo) y tampoco se recibe pasivamente (como en el empirismo), sino que es una construcción que el sujeto va realizando desde su propia realidad y comunicación con sus orígenes, con su comunidad, es decir, con su mundo. Ninguna persona parte de cero en la educación (menos en la universidad), las personas adquieren nuevos conceptos y los elaboran a partir de lo que el estudiante ya posee, de 
tal modo, que el nuevo conocimiento lo relaciona, lo concatena, lo compara con lo que ya sabe. Le adhiere o lo deshecha, lo reajusta a sus estructuras.

2. La capacidad constructiva del alumno es la materia base en el aprendizaje. Este camino requiere de la totalidad del individuo (gestalt desde algunas explicaciones psicológicas. Aunque el mismo Piaget no le diera ese nombre pero se refiere al mismo individuo en su completitud), es decir, sus actitudes, su historia, sus expectativas y sus motivaciones. Por tales razones, el conocimiento se construye a partir de la acción. La praxis permite que la persona lleve a cabo las conexiones entre los objetos del mundo que va experimentando, entre sí mismo y esos objetos, y que al interiorizarse, reflexionarse y abstraerse, conforman el conocimiento. Esta acción puede materializarse físicamente o representarse mentalmente mediante la palabra, el signo o la imagen.

3. Como producto de estas conexiones los individuos generan nuevos esquemas y como parte de resultados nuevos se incide en su práctica cotidiana. Se reflexiona en la generación de nuevos conceptos y se genera una nueva actitud respecto a lo que se entiende por el mundo. Ese saber no se almacena de forma bancaria, en forma sumatoria o de simple acumulación de experiencias de aprendizaje.

4. La dinamicidad de este proceso implica que el avance no siempre es ascendente ni lineal, en ocasiones hay dificultades, incluso equivocaciones, que nos hacen replantear lo aprendido.

Piaget (1970), entendió que el desarrollo cognoscitivo ha de explicarse como un proceso en la relación entre la experiencia que se tiene con la realidad del medio circundante y las estructuras de pensamiento que se van desarrollando a partir de ella para adaptarse al mundo. En este camino, aparece el conflicto cognitivo.

Piaget denomina conflicto cognitivo cuando el individuo analiza el saber que ya posee pues le parece insatisfactorio, de tal forma, se lanza en búsqueda de explicaciones que le satisfagan más. El profesor tiene entre sus funciones provocar este conflicto cognitivo a partir del conocimiento previo de sus estudiantes, cuestionar respetuosamente los conocimientos que se tienen y generar respuestas más complejas. A diferencia de la enseñanza tradicional, el constructivismo no centra 
su esfuerzo en los contenidos sino en el estudiante, en el cambio que el alumno va generando en su propia cosmovisión.

Contrariamente, Vigotsky (1987), ubica a los otros como parte del ambiente que el individuo ha de reconocer y aprender como experiencia sociocultural. De tal forma, que el aprendizaje no puede ser individual, sino en relación. Definió el aprendizaje como fenómeno que ocurre en una "zona de desarrollo próximo" en otras palabras, el individuo puede resolver problemas más complejos en grupo, de los que resolvería solo. Al contrario que Piaget, en el constructivismo social de Vigotsky, el conocimiento, además de formarse a partir de las relaciones ambiente-yo, es la suma del factor entorno. Efectivamente, los conocimientos se forman a partir de los propios esquemas pero además también de su comparación con los esquemas de los demás individuos que lo rodean.

Así, el docente constructivista confía en la capacidad de sus alumnos y enseña a partir de problemas que son significativos para los alumnos. El reconocimiento básico que ha de hacer el docente, implica que los estudiantes son autónomos moral y cognitivamente. Contrario a la concepción colonialista donde se presuponía que los indígenas no tenían la capacidad de crear civilización por sí mismos, por eso era importante imponer una tutela.

En conclusión, las líneas fundamentales del constructivismo señalan que el aprendizaje es un camino activo, no pasivo; por lo tanto, es un conocimiento construido, que se descubre o se inventa socialmente en el proceso de dar sentido al medio, por tanto, se requiere de apertura para asumir los desafíos de la modificación de esquemas. Lo que significa que, los indígenas tienen un sentido riquísimo en experiencias y en cultura, de ninguna manera se ha de pretender eliminar o desechar estos saberes. Efectivamente, los alumnos integran a su visión del mundo los estudios universitarios, y los conjuntan con lo ya sabido, enriqueciendo sensiblemente sus perspectivas, en muchas ocasiones más de lo que otros alumnos mestizos, pues éstos no han tenido que adaptarse a una nueva cultura, mientras que los alumnos indígenas sí. Poder plantear a los alumnos (indígenas y no) el respeto a sus orígenes y saberes familiares y culturales con el fin de ampliar las perspectivas de relación, comunicación y aprendizaje. 
Indudablemente, la realidad de los estudiantes indígenas presenta grandes desafíos, aspecto que se muestra en la segunda parte de este texto, a partir de los resultados de la investigación realizada a los estudiantes indígenas de la UMAR del estado de Oaxaca.

\section{La Universidad del Mar}

La Universidad del Mar (UMAR) es una institución educativa de nivel superior que pertenece al Sistema de Universidades del Estado de Oaxaca (SUNEO). Se encuentra en la zona sur del estado, en la región costa. Fue creada para dar respuesta a la necesidad de acercar los centros especializados a las regiones del estado, y permitir a los jóvenes oaxaqueños cursar una carrera profesional sin tener que trasladarse a la ciudad capital o emigrar a estados vecinos como Veracruz, Chiapas, Guerrero y Puebla. La UMAR abrió sus puertas el 22 de agosto de 1992 en Puerto Ángel, Oaxaca. En 2001, inician actividades los campus Puerto Escondido y Huatulco.

Además de ser una institución de educación superior, es un centro de investigación, en el que tanto profesores-investigadores como alumnos permanecen de tiempo completo dedicados a la docencia y a la investigación. En apoyo a esta formación integral del alumno, la UMAR ha desarrollado su modelo de enseñanza con base en los siguientes planteamientos y exigencias: los alumnos de tiempo completo (sistema escolarizado) con un programa de asistencia obligatoria (más del $80 \%$ ) a clases y a tiempo de estudio en biblioteca, laboratorio de cómputo, conferencias y asesorías. Sistema de Evaluación constante de los alumnos (exámenes parciales, ordinarios, finales, extraordinarios y especiales). Programa de becas, colegiatura ilimitada en número para

quienes tienen problemas económicos y calidad académica, así como un programa limitado de becas alimentarias (UMAR, 2014).

\section{Características generales de los estudiantes indígenas de la UMAR}

La composición de la comunidad estudiantil indígena respecto del género, en su mayoría son mujeres, ya que representan casi el $61 \%$ mientras que los hombres conforman alrededor del 39\%. La permanencia de los estudiantes en la Universidad del Mar se debe a que es la mejor opción para estudiar una licenciatura (42.85\%), el 35\% dijo 
que fue su única opción. Considerando el programa de becas que ofrece la institución el $11 \%$ de los estudiantes indicó que fue un determinante a la hora de solicitar su ingreso y, sólo el 1\% contestó que sus padres los obligaron.

Las razones que motivan a los jóvenes indígenas a cursar una carrera profesional, se encuentra que, el $76 \%$ quiere un mejor futuro (una mejor calidad de vida), el 5.3\% indica que estudia para tener un buen empleo, el 6.8\% dijo que para ayudar a su comunidad de origen, mientras que el $4.5 \%$ lo hace con el fin de adquirir conocimiento y seguir aprendiendo.

Con respecto a la distribución de estudiantes por carrera el $40.9 \%$ cursa la licenciatura en Administración Turística, seguida de Relaciones Internacionales con un $38.3 \%$. El 13.68\% realiza estudios de Economía y una pequeña proporción (6.8\%) Ciencias de la Comunicación.

En relación con el tipo de beca con que cuentan los indígenas universitarios es pertinente mencionar que gran parte de ellos proceden de comunidades rurales con pocas o nulas opciones educativas, provienen de familias de escasos recursos, cuyos padres en la mayoría de los casos no pueden solventar sus gastos universitarios. ${ }^{5}$

Por lo anterior, algunos estudiantes salen de sus comunidades para trabajar y costear sus estudios. Tal es el caso de algunos estudiantes indígenas de la UMAR ya que, el $2.5 \%$ han sido apoyados por el Consejo Nacional de Fomento Educativo (CONAFE), mediante una beca por haber prestado un servicio profesional de enseñanza educativa en otras comunidades. Un porcentaje alto de estudiantes indígenas, el $68.8 \%$ tienen beca colegiatura y el 30.8\% cuenta con el apoyo del Programa Nacional de Becas. (PRONABES) además de tener la beca colegiatura.

Con respecto a los hablantes de lengua indígena, la figura 1 muestra las lenguas y la cantidad de jóvenes hablantes. La lengua étnica más hablada es el zapoteco ${ }^{6}(59.4 \%)$ seguida del Mixteco (9.4\%), Mixe (9.4\%), Chatino (6.3\%), Triqui (6.3\%), Chinanteco (6.3\%) y Mazateco (3.1\%).

5 De acuerdo con INEGI, en el estado de Oaxaca el 52.7\% de la población vive en localidades rurales y el 47.3 en zona urbana. El $98.3 \%$ de las localidades son rurales, cifra superior al promedio nacional.

6 Cabe señalar que gran parte de los estudiantes indígenas que hablan zapoteco provienen de la región del Istmo y Valles Centrales debido a la cercanía al lugar donde está ubicada la institución. 
Figura 1. Estudiantes hablantes de lengua indígena

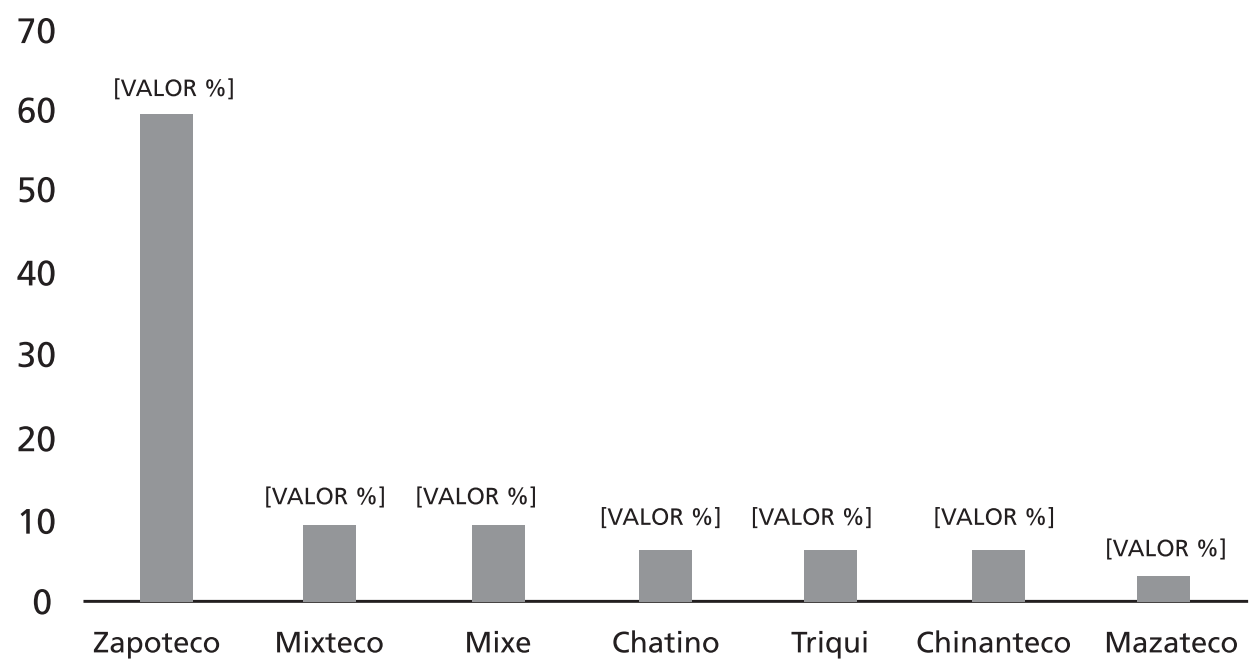

Fuente: Elaborado con datos de investigación de campo 2014.

El $31.7 \%$ de los estudiantes reconoce entender y hablar perfectamente una lengua. Este mismo porcentaje de personas señaló no tener ninguna dificultad con el manejo oral y escrito del español teniendo como lengua materna un idioma indígena. El 39\% lo entiende y habla de forma regular y el $29 \%$ no habla una lengua étnica pero sí lo entiende ya que sus familiares son hablantes.

\section{Problemas a los que se enfrentan los estudiantes indígenas}

Teniendo en cuenta que los estudiantes indígenas provienen de comunidades rurales donde los recursos económicos son escasos y las condiciones educativas son precarias y de mala calidad (con poca o nula infraestructura), es evidente que hay desventajas y diferencias sociales, culturales y académicas entre los estudiantes indígenas y no indígenas dentro de la institución.

En este sentido, existen grandes retos para ellos al llegar a la universidad. Durante su estancia, los indígenas universitarios reconocen una serie de problemáticas y dificultades que se les presenta, así mismo, los lleva a utilizar distintas estrategias para hacerles frente a éstas, incluso en algunos casos los obliga a modificar su conducta. Por ello, en este apartado concentramos el conjunto de problemas de carácter 
personal, académico, social y cultural que mencionaron, y a lo que se enfrentan durante su estancia en la Universidad del Mar.

Los problemas personales mencionados por los alumnos indígenas se muestran en la figura 2. Cerca de la mitad de los indígenas universitarios enfrenta situaciones económicas (49.6\%), lo que tiene una relación directa con el lugar de procedencia qué como bien se dijo, la mayoría es originario de alguna comunidad rural.

La inseguridad personal (19.5\%) y el bajo rendimiento académico (15\%) son otros de los factores más frecuentes a los que se enfrentan los estudiantes. Es probable que los aspectos anteriores estén relacionados con otros problemas mencionados como el de adaptación, timidez y baja autoestima.

El tema de la discriminación hacia las personas indígenas es algo muy usual, sin embargo, pocos son los estudiantes que señalan que exista algún tipo de discriminación de parte de sus compañeros (4.5\%), al igual que el hecho de hablar una lengua indígena; ubicándose por debajo de otros problemas como soledad, pleitos y discusiones con compañeros y la falta de amigos (véase figura 2).

Considerando estos resultados de campo, es posible decir que los problemas de comunicación y convivencia, adaptación y soledad dificultan la interacción con el resto de sus compañeros y profesores, lo que tiene como resultado un bajo desempeño académico. Así, aspectos como la soledad limitan la posibilidad de comunicarse y convivir con otras personas, lo que a su vez genera, o bien, incrementa gran inseguridad personal, hay falta de amigos y compañerismo para desenvolverse correctamente.

Al profundizar en los problemas económicos a los que se enfrentan los estudiantes indígenas se encuentra que el $46.6 \%$ es de escasos recursos monetarios, es decir, provienen de hogares con un nivel de ingresos bajo; el 24.8\% no tiene dinero para comprar libros de apoyo a su formación académica. Otros estudiantes mencionaron que en algunas ocasiones ven limitados sus recursos económicos para poder realizar el pago de renta de su vivienda (18\%) y para comer (17\%) por lo que, tienen una mala alimentación que en algunos casos, conlleva posteriormente a presentar problemas de salud (véase figura 3). 
Figura 2. Problemas personales a los que se enfrentan los estudiantes indígenas

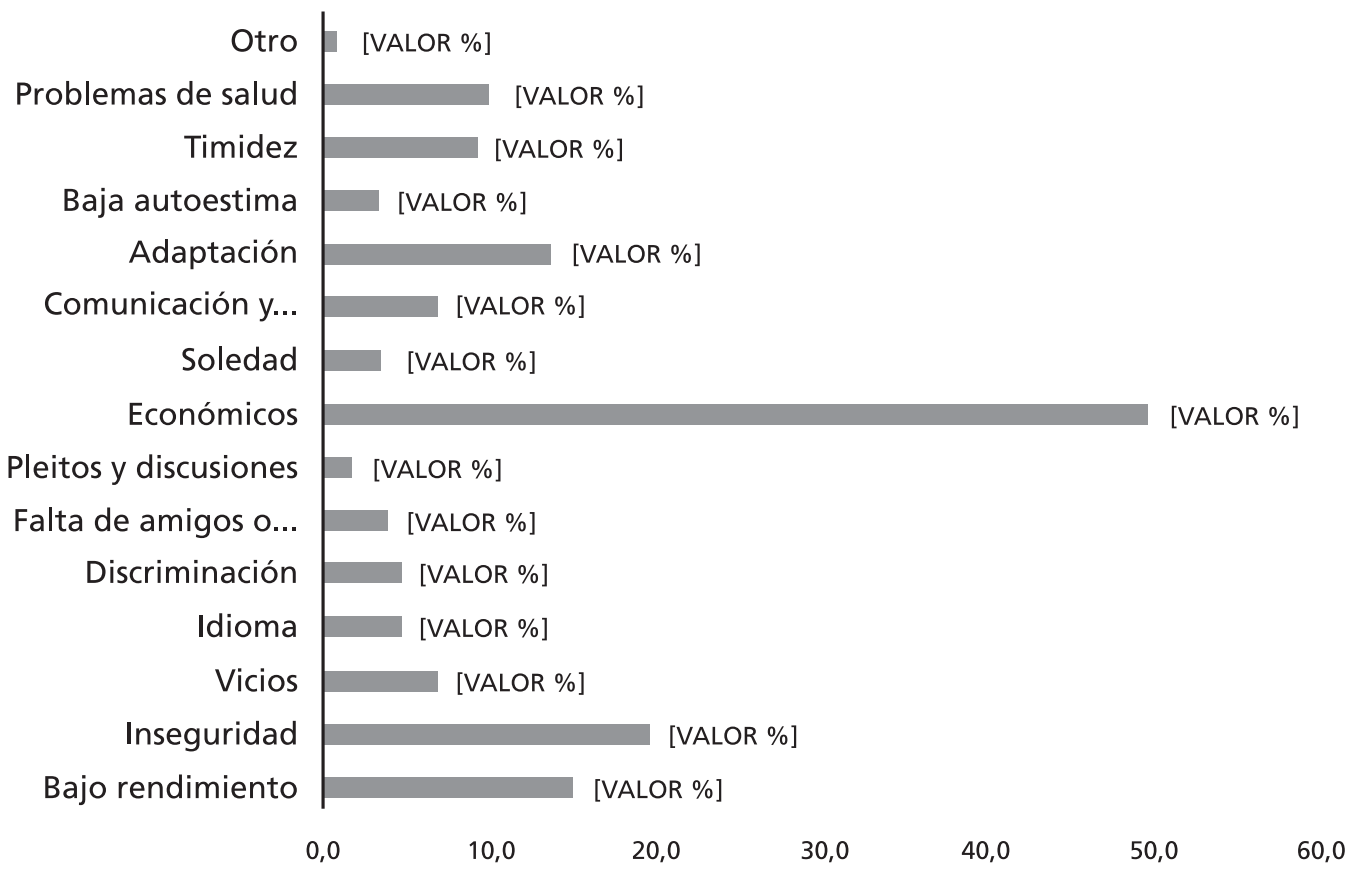

Fuente: Elaborado con datos de investigación de campo 2014.

\section{Figura 3. Problemas económicos a los que se enfrentan los estudiantes indígenas}

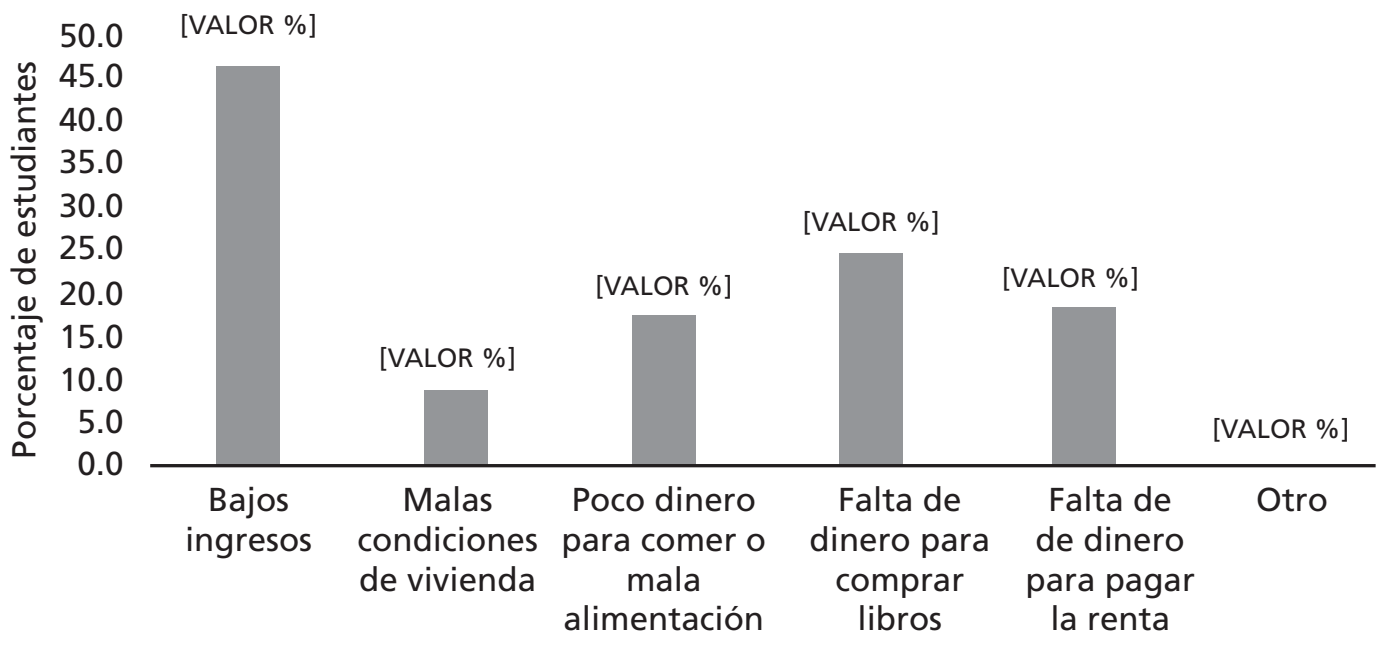

Fuente: Elaborado con datos de investigación de campo 2014. 
$\mathrm{Al}$ preguntarles a los estudiantes indígenas si se sienten diferentes al resto de sus compañeros, el $28 \%$ dijo que sí. Las razones principales se ilustran en la figura 4. El 93.1\% declaró que por la manera distinta de ver la vida (cosmovisión), mientras que el resto (6.8\%) señaló que por la forma en que se viste.

\section{Figura 4. Razones por las que los estudiantes indígenas se sienten diferente a los demás}

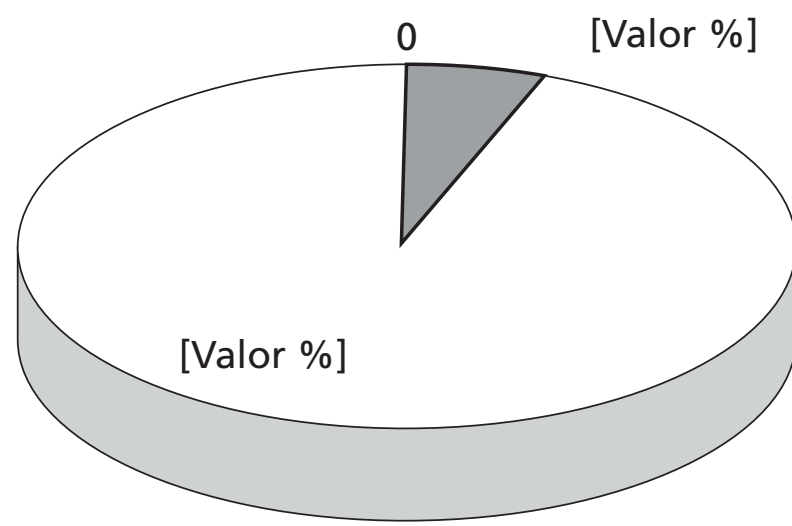

\section{Por su forma de vestir \\ $\square$ Por su forma de ver la vida (cosmovisión)}

Fuente: Elaborado con datos de investigación de campo 2014.

De acuerdo con la Comisión Nacional para el Desarrollo de los Pueblos Indígenas (CDI), la cosmovisión indígena ${ }^{7}$ tiene que ver con la lengua, historia y el medio natural que influye en cada uno de los aspectos de la vida, pues a través de estos elementos se explican los fenómenos naturales, y la manera en que interactúan con la naturaleza, así como, la organización de su vida social y religiosa. En resumen, el término está relacionado con la forma de pensar y ver la vida de una manera diferente.

La importancia de hacer una breve referencia al concepto de cosmovisión con anterioridad, obedece a que éste es mencionado como uno de los problemas culturales a los que se enfrentan los indígenas

7. Definida como la visión que cada uno de los pueblos indígenas tienen del mundo, la concepción de la forma que tiene el cosmos, la naturaleza, los seres humanos así como su orden (Navarrete, 2008. Citado CDI. 
universitarios de la UMAR, como se ve en la figura 5. El 24\% considera que su forma de pensar, ver la vida y actuar diferente aunado al arraigo a las costumbres de las comunidades de origen (9.7\%) han representado un obstáculo para su desarrollo durante su estancia en la universidad.

Otros problemas señalados están vinculados a que los estudiantes indígenas tienen un menor nivel académico, intelectual y cultural (7.3\%), la falta de conocimiento en cuanto al uso de la computadora y la internet $(8.9 \%)$. En menor medida son citados, otros elementos como la religión, el racismo o el idioma.

\section{Figura 5. Problemas culturales a los que se enfrentan los estudiantes indígenas}

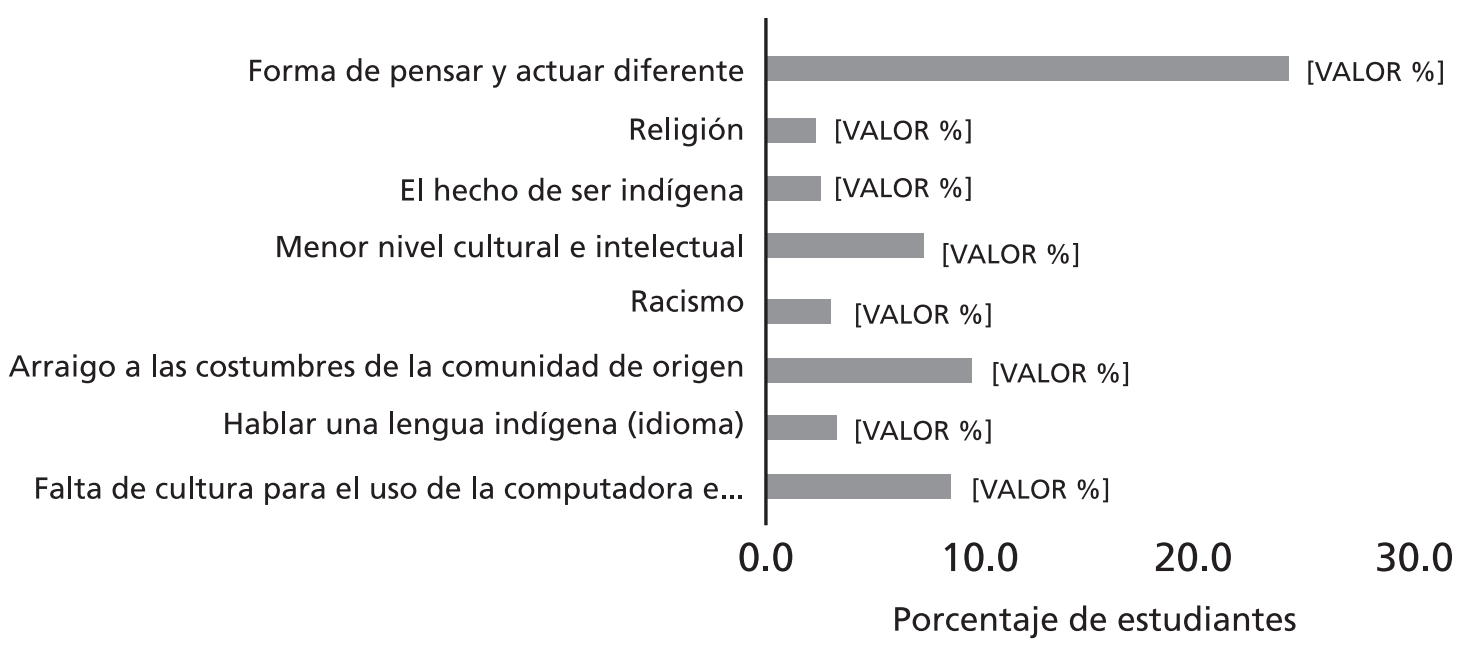

Fuente: Elaborado con datos de investigación de campo 2014.

En relación con los problemas académicos que enfrentan los estudiantes, en la figura 6 , el $28 \%$ no tiene el hábito de leer, lo que conlleva a un lento aprendizaje, el $22 \%$ dijo que no tiene internet en casa, porque lo que suelen retrasarse para realizar las lecturas y tareas.

El $20 \%$ no conoce técnicas de estudio, el $15 \%$ señala que los profesores utilizan un lenguaje complejo a la hora de dar sus clases, por lo que para ellos es difícil comprender. Un porcentaje pequeño (6\%) reconoce tener deficiencias académicas a nivel primaria y secundaria y el $7 \%$ no tiene computadora personal, lo que representa una desventaja hacia el resto de sus compañeros para estar actualizados en términos de conocimiento y aprendizaje. 


\section{Figura 6. Problemas académicos a los que se enfrentan los estudiantes indígenas}

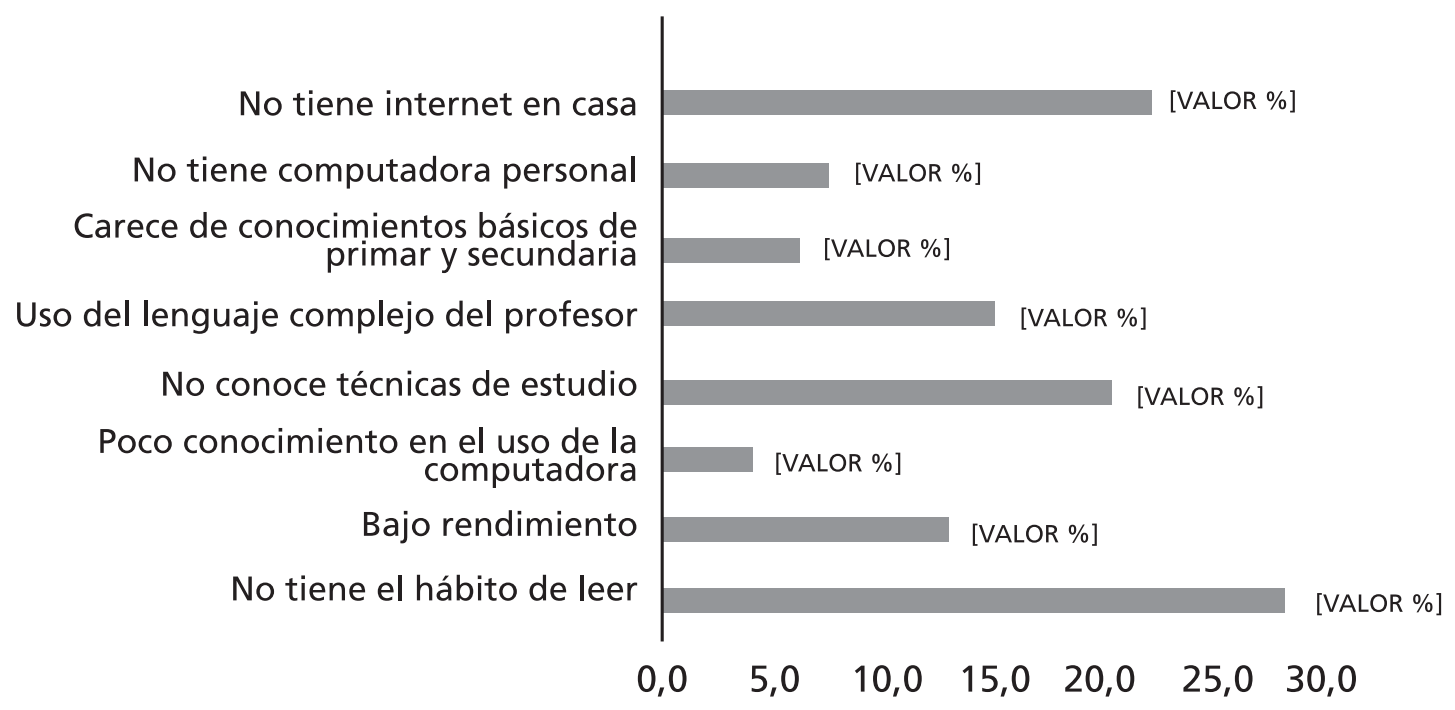

Fuente: Elaborado con datos de investigación de campo 2014.

Finalmente, la figura 7 ilustra los factores sociales vinculados con el entorno en el que se desenvuelven los estudiantes indígenas y que representan un problema, al mismo tiempo, interfieren en el desempeño académico y social. El 15\% declaró tener problemas familiares que tienen que ver con violencia doméstica, vicios, carencia de recursos económicos y enfermedades. Otro porcentaje de estudiantes (7.5\%) dijo que proviene de una familia desintegrada.

El 16.5\% no tiene confianza en sus compañeros de clase, el 14.3 $\%$ dice tener falta de comunicación, mientras que $9.8 \%$ de los estudiantes indígenas presentan dificultad para relacionarse con sus compañeros; estos aspectos resultan ser un obstáculo para establecer relaciones sociales. Otros inconvenientes mencionados son discriminación por parte de sus compañeros $(2.8 \%)$ y por parte de los maestros (3.0\%). 
Figura 7. Problemas sociales a los que se enfrentan los estudiantes indígenas

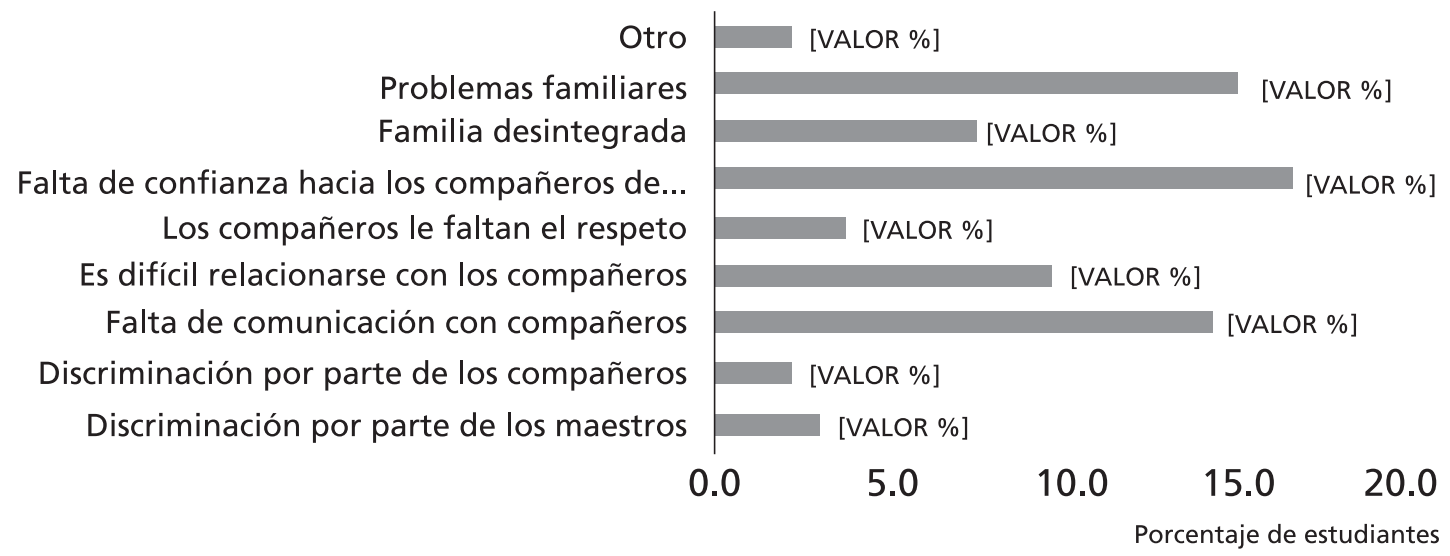

Fuente: Elaborado con datos de investigación de campo 2014.

\section{Conclusiones}

En este artículo se ha transitado el siguiente derrotero: en primer lugar, una suscinta descripción de la realidad indígena que se vive en México. Esta descripción permitió visualizar en datos duros (ofrecidos por el INEGI en su mayoría) el desafío que implica la educación superior a esta población.

Lo que nos llevó al siguiente paso: ¿Qué tipo de educación ha de desarrollarse? El constructivismo dio la pauta para dar una respuesta. Debido al reconocimiento que hace del saber previo, el constructivismo plantea un punto de partida incluyente y propositivo de construcción del saber, alejado de los dos extremos que en ocasiones han radicalizado la enseñanza, a saber: que se ha de dejar de lado el conocimiento previo para dar paso al nuevo saber; o bien, que no se puede aprender nada nuevo y entonces hay que abandonar la empresa de la educación. El constructivismo demuestra que ambas posturas son erróneas y que se puede llevar a cabo una educación que considere los factores de origen y el pensamiento crítico para construir nuevos saberes.

Después de haber planteado esta breve discusión, el siguiente paso fue el caso práctico, concretamente los alumnos indígenas que 
cursan algún grado escolar en la Universidad del Mar, cuyas conclusiones de las encuestas son las siguientes:

1. Hay más mujeres que hombres, lo que se podría interpretar de la siguiente manera: contrario a lo que comúnmente se piensa sobre el poco apoyo hacia las mujeres en términos de estudio, la realidad en la UMAR-Huatulco es que las familias indígenas han optado por apoyar el estudio profesional de las mujeres.

2. De las cuatro carreras que se imparten en el campus, $40.9 \%$ han elegido Administración Turística, que es la carrera de mayor demanda en el campus, sin embargo, escasamente eligen Ciencias de la Comunicación, sin ser esta carrera la menos demandada, en general, probablemente por el estigma de que esta carrera es cara en los materiales que se usan.

3. La mayoría de los estudiantes (65.8\%) percibe la beca otorgada por la propia universidad, que les exime del pago de colegiatura, pero que no les otorga dinero en efectivo. Mientras que el $30.8 \%$ percibe la beca Pronabes. Esta beca puede ser recibida al mismo tiempo que la beca colegiatura y consiste aproximadamente en mil pesos mensuales, cantidad realmente baja para las exigencias de una universidad de tiempo completo.

4. Por la región en que se encuentra la universidad, la mayor parte de los estudiantes indígenas son zapotecos $(59.4 \%)$, provenientes de las comunidades costeñas e istmeñas.

5. En la percepción general interpretan que estudiar una carrera universitaria les proveerá de un mejor futuro $(76.5 \%)$ y en segundo lugar, que podrán así ayudar a su comunidad (6.8\%).

6. Los problemas personales a que se enfrentan son sin lugar a dudas la solvencia económica (49.6\%) y curiosamente el sentimiento de inseguridad (19.5\%).

7. Se reconocen diferentes en su pensar y actuar (24.2) y fuertemente arraigados a sus costumbres (9.7\%), ambos datos los ubican como dificultades culturales en la adaptación a la universidad.

8. Como problemas académicos reconocen la falta del hábito de leer (28.2\%) y la ausencia de técnicas de estudio (20.6\%), así como dificultades para entender el lenguaje técnico de los profesores $(15.3 \%)$. 
9. Refieren como un problema social la falta de confianza para sus compañeros de grupo (16.5\%), la falta de comunicación con sus compañeros $(14.3 \%)$ junto con problemas familiares (15\%).

10. Los bajos ingresos prevalecen entre los problemas económicos que refieren (46.6\%) y la dificultad para adquirir los libros (24.8\%), dificultades para pagar la vivienda (18\%), seguido de un aspecto sumamente importante: poco dinero para comer o mala alimentación $(17.3 \%)$.

11. $93.1 \%$ se siente diferente al resto de sus compañeros por su interpretación de la vida (cosmovisión).

12. Finalmente, los estudiantes han elegido esta universidad porque perciben que es la mejor opción (42.8\%), a la vez que, 35.33\% refieren que es la única opción.

Esta investigación nos ha brindado un diagnóstico que permite observar los retos a los que se enfrentan los estudiantes indígenas en la universidad, principalmente se resumen en económicos, académicos y personales. Muestra una realidad que es difícil reconocer y asumir, al mismo tiempo, no es considerada ni por las instituciones ni por los docentes, pero que es fundamental y debe ser tomada en cuenta para transformar el esquema educativo.

Para los docentes es un doble desafío ya que por una parte estando inmersos en el ámbito académico y de la investigación son responsables de seguir explorando este tema y difundirlo; y por otra, de manera directa son parte necesaria en la formación de los estudiantes indígenas, por ello, es importante proponer y generar proyectos que coadyuven al desarrollo de los estudiantes y a la superación de estas vicisitudes.

Este apenas es un acercamiento a las situaciones relacionadas con los estudiantes indígenas y su entorno durante su formación académica a nivel superior. Queda pendiente un estudio posterior sobre la eficiencia terminal académica, de estos estudiantes, lo cual implica un estudio longitudinal y de seguimiento más amplio. 


\section{Referencias bibliográficas}

Asociación Nacional de Universidades e Instituciones de Educación Superior (2010). Anuario estadístico 2010-2011: Población escolar de licenciatura en universidades e institutos tecnológicos. México: ANUIES.

------- (2010), Programa de Apoyo a Estudiantes Indigenas en Instituciones de Educación Superior. Recuperado de http://paeiies. anuies.mx/public/index.php?pagina $=$ cobertura.html

Barreno, L. (2003). Educación superior indígena en América Latina: La educación superior indígena en América Latina. Caracas: Unesco-Iesalc.

Bonfil Batalla, G. (1987), México profundo: una civilización negada. México: Grijalbo.

Carbó, T. (1982), Los indígenas, el estado y la educación en Revista Latinoamericana de estudios educativos, CIESAS, México.

Chávez Arellano, M. (2008), Ser indígena en la educación superior ¿desventajas reales o asignadas? Revista de la educación superior, 37, 31-55. Recuperado de http://www.scielo.org.mx/scielo. php?script=sci_arttext\&pid=S0185-27602008000400003\&ln$\mathrm{g}=$ es\&tlng=es.

Comisión Nacional de los Pueblos Indígenas y Programa de las Naciones Unidas para el Desarrollo (2006). Informe sobre desarrollo humano de los pueblos indígenas. México.

Garzón, C. y Vivas, M. (1999). Una didáctica constructivista en el aula universitaria. Revista Educere, 3 (5).

Instituto Nacional De Estadística, Geografía e Informática (2010). Censo de Población y Vivienda. México. Recuperado de http:// www.inegi.org.mx/est/contenidos/proyectos/ccpv/cpv2010/ Default.aspx

López Cárdeña, D. y Viveros Álvarez, R. (2003). Educación intercultural y pueblos indígenas en México. Elementos para analizar nuevas metáforas del siglo XXI. México.

Navarrete Linares, F. (2008). Los pueblos indígenas de México. México: CDI. Recuperado de http://www.cdi.gob.mx/index. php?option $=$ com_content\&view $=$ article $\&$ id $=272 \&$ Itemid $=58$ 
Padilla Arias, A., (2002). El doble carácter de la educación indígena: reproducción y resistencia en Reencuentro, UAM-Xochimilco, P. 40-52.

Parsons, T. (1975). "Some theoretical considerations on the nature and trends of change of ethnicity", en GLAZER, Nathan y MOYNIHAN, Daniel P., 1975, Ethnicity. Theory and Experience, EUA, Harvard University Press.

Piaget, J. (1970). Genetic epistemology. New York: W. W. Norton and Company.

Shmelkes, S. (2003). Educación superior intercultural. El caso de México. Conferencia dictada en el Encuentro Internacional Intercambio de Experiencias Educativas, "Vincular los Caminos a la Educación Superior", organizado por la Ford Foundation, la Unidad de Poyo a las Comunidades Indígenas de la Universidad de Guadalajara y la ANUIES, los días 17 a 19 de noviembre de 2003.

UNESCO-IBE (2010). Datos Mundiales de Educación. 7a edición, 2010/11. Recuperado de http://www.ibe.unesco.org/

Vigotsky, L. S. (1987). Historia del desarrollo de las Funciones Psíquicas Superiores, Ed. Científico Técnica. Ciudad de la Habana, Cuba. 